\title{
Tracking within-category colors is easier: Color categories modulate location processing in a dynamic visual task
}

\author{
Mengdan Sun ${ }^{1} \cdot$ Luming Hu ${ }^{1}$. Lingxia Fan ${ }^{1} \cdot$ Xuemin Zhang ${ }^{1,2,3}$ \\ Published online: 5 July 2019 \\ (C) The Psychonomic Society, Inc. 2019
}

\begin{abstract}
The categorical perception (CP) of color describes the phenomenon that colors across categories (e.g., blue and green) are more discriminable than within-category colors (e.g., green) even when the perceptual distance is controlled. While most studies are conducted in a static visual scene, the current study investigated the effect of color categories when tracking multiple colored objects within two experiments. The targets or distractors were either from the same color category or from two different categories, and the perceptual distance was controlled across all conditions. We found that location tracking was facilitated when the targets or the distractors shared the same color category in the tracking task, which required location tracking and color memorization at the same time, compared with when they were from two different categories (Experiment 1). By contrast, when location tracking was the sole demand and no explicit color processing was required, the target $\mathrm{CP}$ effects, but not the distractor $\mathrm{CP}$ effects, persisted (Experiment 2). We conclude that color categories can dynamically modulate preattention visual processing when color working memory is involved, but that attention is necessary to produce CP effects when color is irrelevant to the task.
\end{abstract}

Keywords Color $\cdot$ Categorical perception $\cdot$ Tracking $\cdot$ Language $\cdot$ Memory

\section{Introduction}

Categorical perception (CP) describes the tendency to perceive the visual world in terms of our learned categories (Harnad, 1987). For instance, when we perceive colors, we tend to classify them into discrete categories, such as "red" or "green," despite the continuous nature of wavelengths related to colors. Categorical perception of color suggests that individuals are faster or more accurate at discriminating colors from two different categories (e.g., blue and green) than from the same category

Electronic supplementary material The online version of this article (https://doi.org/10.3758/s13421-019-00959-9) contains supplementary material, which is available to authorized users.

Xuemin Zhang

xmzhang@bnu.edu.cn

1 Beijing Key Laboratory of Applied Experimental Psychology, National Demonstration Center for Experimental Psychology Education (Beijing Normal University), Faculty of Psychology, Beijing Normal University, Beijing 100875, China

2 State Key Laboratory of Cognitive Neuroscience and Learning and IDG/McGovern Institute for Brain Research, Beijing Normal University, Beijing, China

3 Center for Collaboration and Innovation in Brain and Learning Sciences, Beijing Normal University, Beijing, China (e.g., green) when the chromatic distance is controlled (Drivonikou et al., 2007; Franklin et al., 2008; Gilbert, Regier, Kay, \& Ivry, 2006; Roberson, Pak, \& Hanley, 2008; Winawer et al., 2007). A range of visual tasks, including judged similarity (Kay \& Kempton, 1984) and visual search (Daoutis, Pilling, \& Davies, 2006; Lindsey et al., 2010), have been used to demonstrate the unique effect of color categories.

To the best of our knowledge, most studies of CP effects have used instantly presented visual inputs, whereas the realworld scene is filled with continuous and dynamic stimuli. For example, when walking on the street, we often track the movement of multiple cars and pedestrians in colors for a relatively long duration. Understanding how we encode continuously moving colors or colored objects is important. Thus, our previous study explored CP effects in a dynamic task termed "multiple object tracking" (MOT; Sun, Zhang, Fan, \& Hu, 2018). In a typical MOT task (Cavanagh \& Alvarez, 2005; Meyerhoff, Papenmeier, \& Huff, 2017; Pylyshyn \& Storm, 1988; Scholl, 2009), participants are presented with multiple objects with identical or heterogeneous surface features, and a subset that is cued as targets. After cueing, all of the objects start to move randomly and independently for a period of time during which participants need to keep track of the targets among the distractors. When the objects stop moving, the participants are required to report the location of each target. 
In our previous study (Sun et al., 2018), the participants were required to track four targets among four distractors, and the targets and distractors were either from one same category (green or blue) or from two different categories (green and blue) when controlling for the hue distance $\left(5^{\circ}, 10^{\circ}, 20^{\circ}, 30^{\circ}\right.$, or $\left.35^{\circ}\right)$. The results showed that the color categories did not affect the tracking performance and that the target-distractor hue distance was the dominant factor in determining the tracking performance. The absence of CP effects surely contradicted the idea that color space was permanently altered by the acquisition of color categories (Harnad, Hanson, \& Lubin, 1991) or that color vision itself was inherently categorical (Regier, Kay, \& Khetarpal, 2007). However, this finding does not suggest that $\mathrm{CP}$ effects cannot occur at all during MOT, because a broad sense of $\mathrm{CP}$ includes category effects at more cognitive levels of processing, such as attention and subjective evaluation (Witzel, 2018). From this perspective, the presence of $\mathrm{CP}$ effects depends on the nature of specific tasks (Davidoff, 2015; Webster \& Kay, 2012). The task in our previous study encouraged low-level processing, because perceptual grouping based simply on the target-distractor hue distance could be formed automatically. When the tracking procedure changed, whether color categories would be able to modulate the tracking performance warranted exploration. Building upon the previous study (Sun et al., 2018), the current study altered several aspects of the tracking procedure within Experiment 1, which were expected to promote CP effects.

In the current study, we differentiated color categories within both the targets and distractors. Instead of sharing one identical hue, such as in Sun et al. (2018), the target-distractor set itself had two different hues that were either within category or cross category. Attention is voluntarily allocated toward targets during tracking (Cavanagh \& Alvarez, 2005; Huang, Mo, \& Li, 2012; Scholl, 2009), and perceptual grouping based simply on the target-distractor hue distance has been shown to form automatically and promote the maintenance of attention toward the targets in the previous study. Hence, participants may not attend to the categorical distinction between the targets and the distractors under the cross-category condition. According to some CP studies, the categorical perception of color may arise from attention (He, Witzel, Forder, Clifford, \& Franklin, 2014; Witzel \& Gegenfurtner, 2015). For instance, Witzel and Gegenfurtner (2015) suggested that CP effects occurred when participants paid attention to the categorical distinction, but not when they made an automatic judgment based on sensory feed-forward information (Witzel \& Gegenfurtner, 2015). Accordingly, the categorical distinction of the targets would be attended to produce potential $\mathrm{CP}$ effects in the current study. In contrast, CP effects of the distractors were much less likely to occur, because generally they were out of attentional foci during tracking.

The color assignment of the moving objects (two colors for the targets and two colors for the distractors) in this study also increased the tracking difficulty, because the targets or the distractors could no longer be easily grouped. In this case, participants may use the category information during tracking to segment the targets from the distractors. Kay and Kempton (1984) proposed the name strategy to explain the CP effects in their odd-one-out task, in which participants might adopt the lexical classification to make a judgment. One of the facilitating conditions to induce this strategy is that the original judgment is hard to make in some sense (Kay \& Kempton, 1984). Thus, the settings of the current study, in which forming segmentation of the objects based on a physiological code is difficult, may promote a categorical way of seeing (Davidoff, 2015; Kay \& Kempton, 1984).

Furthermore, this study asked participants to memorize each target's color in addition to tracking its location and to report the target's identity after tracking. Unlike the previous study, in which the color identity of the objects was irrelevant, the current study required mandatory location-identity binding and maintenance of the color identity in the working memory (Oksama \& Hyona, 2008). The introduction of these late processes may induce consequent $\mathrm{CP}$ effects the same way as many studies using the short-memory two-alternative forced-choice (2AFC) task (Franklin, Clifford, Williamson, \& Davies, 2005; Roberson \& Davidoff, 2000). The short-memory 2AFC task is commonly used to investigate color categories and has found substantial evidence of CP effects (Roberson \& Davidoff, 2000). Researchers proposed that the $\mathrm{CP}$ effects in memory resulted from the use of a categorical or verbal code when perceptual information was not available (Hanley \& Roberson, 2011; Roberson \& Davidoff, 2000; Roberson \& Hanley, 2009, 2010). The requirement of memorizing the hues of four targets in addition to tracking their locations was challenging, and there was a great chance that the participants would take advantage of the labels of the targets for the purpose of more easily retaining the targets' identities. Under this circumstance, tracking targets sharing the same label may be easier than tracking those with different labels, thereby producing $\mathrm{CP}$ effects.

In summary, the current study included the above changes to the tracking task within Experiment 1, which explored the effects of the targets' and distractors' color categories on the tracking performance. We found significant $\mathrm{CP}$ effects suggesting that tracking within-category targets was easier than tracking cross-category targets. The effects were also observed for the distractors. To examine whether the mandatory memorization of the targets' color identities was necessary for production of the $\mathrm{CP}$ effects, we conducted Experiment 2, in which the participants only needed to track the targets' locations, and their color identities were irrelevant. The withincategory benefit was still present in Experiment 2, but only for the targets. Altogether, our study suggested that color categories could dynamically modulate preattention visual processing when color working memory was involved, but that attention was necessary to produce $\mathrm{CP}$ effects when color was irrelevant to the task. 


\section{Experiment 1}

The first experiment adopted a tracking paradigm in which the observers needed to track four colored targets among four distractors and to report both the location and identity information for the targets. The colors of the targets were either from the same category (green) or from two different categories (blue and green). The same manipulation was made to the distractor set. Therefore, four conditions were constructed: target within-category condition, target cross-category condition, distractor within-category condition, and distractor crosscategory condition. The effects of the target-distractor color categories on both the location and identity accuracy were examined.

\section{Method}

\section{Participants}

Seventeen students (six males, ages $21.13 \pm 2.73$ years old) from Beijing Normal University participated for monetary rewards. The participants reported normal or corrected-tonormal vision and normal color vision.

\section{Equipment}

All displays were programmed in MATLAB R2013b (The MathWorks) with PsychToolbox (Brainard, 1997; Pelli, 1997). Stimuli were displayed on a 17 -inch CRT monitor with a resolution of 1,024 $\times 768$ pixels and a refresh rate of $85 \mathrm{~Hz}$. The participants were seated approximately $57 \mathrm{~cm}$ away from the monitor, and $1 \mathrm{~cm}$ on the screen subtended approximately $1^{\circ}$ of visual angle. The monitor was calibrated to a D65 white point ( $x=0.31271, y=0.32902$ in CIE 1931 color space) with the DataColor Spider5 Colorimeter. The lab environment, including the lighting condition and the position of the monitor, was kept unchanged during the whole experiment.

\section{Stimuli and design}

The color stimuli used for the tracking task were chosen from the CIELAB space $\left(L^{*}=70, a^{*}=0, b^{*}=0\right.$, radius of 38$)$ and varied only in hue. A preliminary color-naming task was performed by seven participants who did not participate in the subsequent experiments to determine the green-blue boundary. In this task, 10 colors (from $160^{\circ}$ to $205^{\circ}$ in steps of $5^{\circ}$ ) were presented individually to the participants. The participants needed to respond "green" or "blue" by pressing the "F" or "J" key, respectively. The "blue" response rate of each participant in the color-naming task was fitted with a sigmoid function defined as $1 /(1+\exp (-(\mathrm{x}-\alpha) / \beta))$, where $\alpha$ was the threshold (estimated value at which "blue" would be reported half of the time), that is, the green-blue boundary in our study.
The average subjective green-blue boundary was $185.66^{\circ}$ ( $S D$ $=3.18^{\circ}$ ). Five colors were then selected for the tracking task with angles of $110^{\circ}, 140^{\circ}, 170^{\circ}, 200^{\circ}$, and $230^{\circ}$. These colors were sampled for the moving objects during tracking according to the trial's condition. The moving stimuli were eight disks $\left(\right.$ diameter $=16$ pixels, visual angle: $0.64^{\circ}$ ) presented on a black (RGB: $0,0,0)$ background with a white border $\left(800 \times 600\right.$ pixels, visual angle: approximately $32^{\circ} \times 24^{\circ}$; see Fig. 1).

Experiment 1 had four conditions (target within-category condition, target cross-category condition, distractor withincategory condition, and distractor cross-category condition). Under each condition, two hues were assigned to the four targets, and two other hues were assigned to the four distractors (see Table 1). Thus, every two targets-distractors shared one hue. Under the target within-category condition, the targets had two different green colors $\left(140^{\circ}\right.$ and $\left.170^{\circ}\right)$. Under the target cross-category condition, the targets had one green color and one blue color $\left(170^{\circ}\right.$ and $\left.200^{\circ}\right)$. Under both of the conditions, one of the distractor colors was $30^{\circ}$ greener than the greener target color, and the other distractor color was $30^{\circ}$ bluer than the bluer target color (see details in Table 1). We then swapped the target colors with the distractor colors under the target within-category condition and the target cross-category condition for the objects under the distractor within-category condition and the distractor crosscategory condition, respectively. Notably, the distractors in the two target conditions were both cross category, and the targets in the two distractor conditions were also from two categories. Moreover, the hue differences between the two target colors, between the two distractor colors, and between the target set and the distractor set were equal across the four conditions.

\section{Procedure}

The tracking task consisted of 120 trials that were divided randomly and evenly into four conditions. At the beginning of each trial, eight colored disks were randomly placed inside the white frame, four of which were cued by red boxes for $2 \mathrm{~s}$ to inform the participants that these were the targets to be tracked (cueing phase; see Fig. 1). Two of the targets sampled one hue, and the other two sampled another hue according to the condition. The hues of the distractors were sampled similarly. All then disks started to move independently and randomly at an average speed of 23.04 degrees/s (tracking phase). The objects bounced off the edge of the frame or repelled one another during movement. After a random duration of 4-6 s, the objects stopped moving and were covered by a multicolor square mask for $250 \mathrm{~ms}$ (masking phase). After the mask was removed, all disks reappeared in white (responding phase). At the same time, 15 colored disks appeared above the frame, the hues of which ranged from $100^{\circ}$ to $240^{\circ}$ in steps of $10^{\circ}$. 


\section{Data analysis}

We analyzed two dependent measures: location accuracy and identity error. The location accuracy was calculated as the proportion of correctly tracked targets. The identity error described the ability to bind a hue to a specific target and was calculated as the absolute difference between the actual hue and the response hue when the participants correctly selected a target. Therefore, the identity error was independent of the location accuracy. These two measures allowed us to separate two processes (location tracking and identity memorization). Importantly, although we manipulated the color categories of both the targets and the distractors, the dependent measures were limited to the targets. In other words, the location accuracy or identity error of the distractors was never tested.

To examine the effect of both the target and the distractor categories, a 2 (category: within, category) $\times 2$ (object: target, distractor) repeated-measures ANOVA was carried out separately for the location accuracy and identity error. The main effect of color category and the interaction between category and object were the main concerns in our study and suggested the presence or absence of CP effects and whether the CP effects were modulated by object (target-distractor). The main effect of object, caused by the exchange of color stimuli between the targets and the distractors, was not addressed in detail because of its irrelevance to this study.

\section{Results and discussion}

Both the location accuracy (labeled Loc ACC) and identity error (labeled ID Error) are plotted in Fig. 2. For the location accuracy, the main effects of category and object were both significant-category: $F(1,16)=56.02, p<.001$, partial $\eta^{2}=$ 0.78 ; object: $F(1,16)=34.81, p<.001$, partial $\eta^{2}=0.69-$ but the interaction effect was not significant, $F(1,16)=1.81, p=$ .197 , partial $\eta^{2}=0.10$. This result suggested the presence of
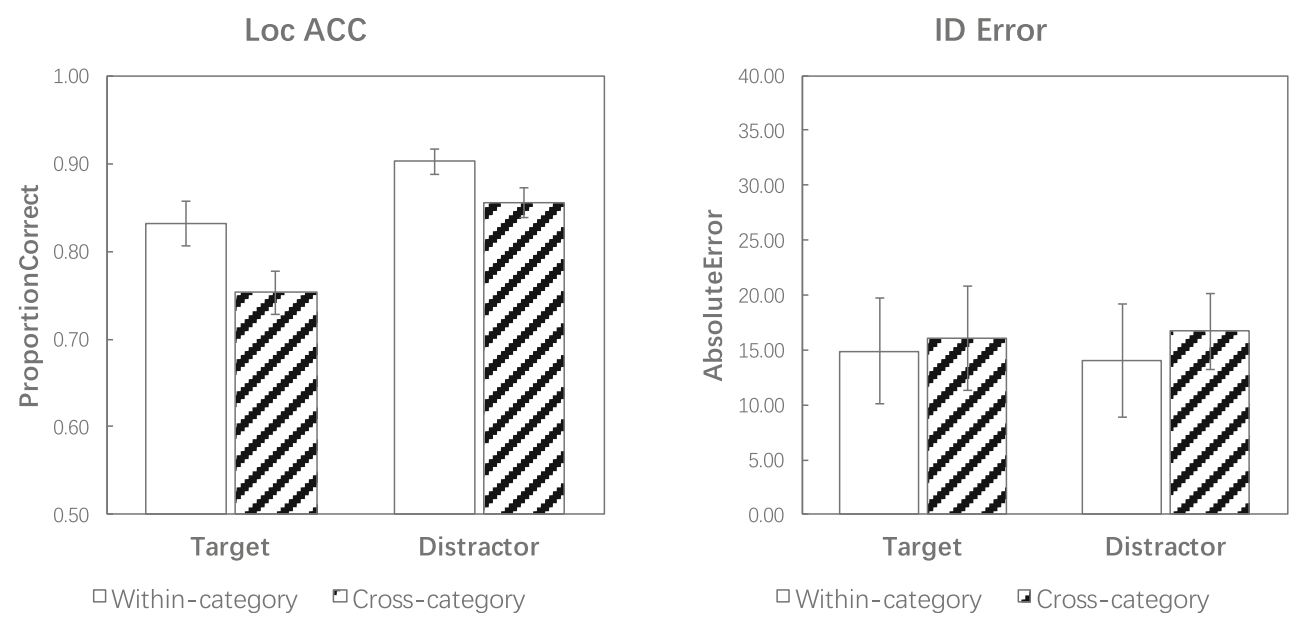

Fig. 2 Mean tracking performance $( \pm 1 S E)$ under the four conditions in Experiment 1 (left panel: location accuracy; right panel: identity error)

CP effects in both the targets and distractors, which allowed the participants to perform better when the targets-distractors were from the same category than when they were not. The main effect of object suggested that the tracking performance was superior under the two distractor conditions than under the two target conditions.

For the identity error, the main effect of category reached marginal significance, $F(1,16)=3.87, p=.067$, partial $\eta^{2}=$ 0.20 , whereas no significant effects were found for object, $F(1,16)=0.018, p=.896$, partial $\eta^{2}=0.001$, or the interaction, $F(1,16)=0.73, p=0.407$, partial $\eta^{2}=0.04$. Since the $p$ value (0.067) was ambiguous, we also adopted the Bayesian framework to assess the effect of color category. A Bayesian repeated-measures ANOVA using JASP (Marsman \& Wagenmakers, 2017) revealed that the category model was slightly more favored than the null model $\left(B F_{10}=1.47\right.$, the ratio between the marginal likelihoods of the alternative model and the null model). Therefore, we suggest here that color categories slightly influence ID error during tracking and that memorizing within-category colors is easier than memorizing cross-category colors.

The color-naming task showed that the average subjective green-blue boundary was $188.94^{\circ}\left(S D=3.28^{\circ}\right)$, suggesting that the categorical membership of color stimuli was appropriate for these participants on average. We provide the subjective green-blue boundary of each participant in the Supplementary Material (see Table S1), which demonstrates that the classification of color stimuli into within category and cross category is also correct for each participant.

These results suggested the presence of CP effects for both the targets and the distractors in the tracking task. Not only was tracking within-category targets easier than tracking cross-category targets (target $\mathrm{CP}$ effects), but the locationtracking process was also affected by whether the distractors were from the same color category or not (distractor CP effects). We hypothesized that the internal representational 
difference was reduced for the within-category colors compared with that for the cross-category colors and that the organization of the objects during tracking was modulated by their color categories. The within-category targets or distractors were grouped more easily. This finding was consistent with the conceptual grouping effect found in the visual search, in which a search among conceptually homogeneous targets was faster and more efficient than a search among conceptually heterogeneous targets (Lupyan, 2008).

To test this hypothesis, we analyzed the representational differences between the two target colors after tracking. Under each condition, two different hues were included for the targets (e.g., $140^{\circ}$ and $170^{\circ}$ ), which are denoted here as Hue 1 and Hue 2. Then, we computed the difference between the average response for Hue 1 and Hue 2, which is labeled as the estimated difference. For example, if a participant responded $130^{\circ}$ on average for the $140^{\circ}$ target hue and $180^{\circ}$ on average for the $170^{\circ}$ target hue (since there were two targets sharing one hue, the response for one particular hue should be a mean), then the estimated difference would be $180^{\circ}-130^{\circ}=50^{\circ}$ (the actual difference was $170^{\circ}-140^{\circ}=$ $30^{\circ}$ ). Figure 3 presents the average estimated differences under the four conditions. Paired $t$ tests revealed that the estimated difference of the hue pair was significantly smaller for the within-category targets than for the cross-category targets, $t(16)=3.17, p=.006$, Cohen's $d=0.77$. On the other hand, when the distractors were from the same category, the difference between the two target hues was estimated to be significantly larger, $t(16)=5.72, p<.001$, Cohen's $d=1.39$, than when the distractors were from two different categories. This

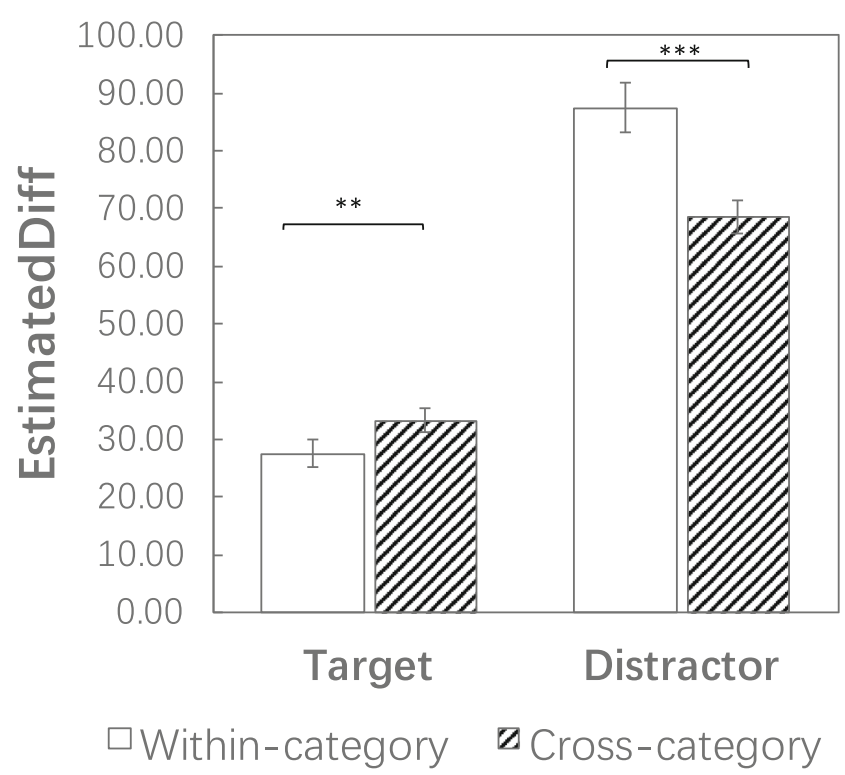

Fig. 3 Mean estimated differences $( \pm 1 S E)$ between the two target colors under the four conditions. The actual hue difference under the target within-category condition and the target cross-category condition was $30^{\circ}$, but $90^{\circ}$ under the distractor within-category condition and the distractor cross-category conditions result suggested that the color categories altered the representational distance between the two target hues during response. When we manipulated the target categories, the withincategory colors were represented as being more similar than the cross-category colors. Furthermore, when the distractors were from the same color category, the two colors of the targets were represented as being more distant from one another than when the distractors were from two categories. Both methods could ease grouping of the targets under the two within-category conditions, and consequently facilitate tracking.

To summarize, Experiment 1 revealed CP effects on tracking when the tracking procedure was altered, which was different from the findings of our previous study (Sun et al., 2018). Furthermore, the presence of CP effects seemed to require minimal attention, because distractor $\mathrm{CP}$ effects were also present. Based on Experiment 1, we intended to examine whether memorizing each target's color identity was necessary to produce $\mathrm{CP}$ effects or whether the current color assignment of the objects alone was sufficient to induce the engagement of color categories. Hence, we conducted Experiment 2, in which the participants were not required to memorize the targets' identities.

\section{Experiment 2}

Experiment 2 aimed to assess whether mandatory memorization of the object identity was necessary for the ability of the color categories to affect location tracking. In this experiment, the participants only needed to track the targets' locations, and the color identity was irrelevant. The categorical effect could be eliminated or weakened because of a lack of voluntary processing of color identities.

\section{Method}

\section{Participants}

Seventeen students (seven males, ages $21.47 \pm 2.24$ years old) from Beijing Normal University participated for monetary rewards. The participants reported normal or corrected-tonormal vision and normal color vision.

\section{Equipment, stimuli, and design}

The equipment, stimuli, and design were identical to those of Experiment 1.

\section{Procedure}

The procedure of Experiment 2 was identical to that of Experiment 1, except for the task requirement. At the 
beginning of each trial, the participants were instructed to track four targets. After tracking, the participants were required to report only the location of the four targets by clicking on them.

\section{Results and discussion}

Figure 4 presents the location-tracking accuracy of Experiment 2. A two-way repeated-measures ANOVA showed that both category and object had significant effects on the Loc ACC - category: $F(1,16)=6.88, p=.018$, partial $\eta^{2}=0.30 ;$ object: $F(1,16)=5.84, p=.028$, partial $\eta^{2}=0.27$ and that the effect of the interaction between them was also significant, $F(1,16)=4.82, p=.043$, partial $\eta^{2}=0.23$. We conducted a simple effect analysis to reveal the CP effects separately for the targets and the distractors. The analysis showed that the Loc ACC was greater in the target withincategory condition than in the target cross-category condition $(p=.003,95 \%$ CI $[0.019,0.075])$, but that no significant differences existed between the distractor within-category condition and the distractor cross-category condition $(p=$ $.456,95 \% \mathrm{CI}[-0.019,0.041])$. The color-naming task showed that the average subjective green-blue boundary was $188.46^{\circ}$ $\left(S D=3.67^{\circ}\right)$. Each participant's subjective boundary is provided in the Supplementary Material (see Table S2) and shows that the classification of color stimuli into within category and cross category is appropriate for each participant.

The results of Experiment 2 showed that CP effects still occurred in the tracking task when the participants were not explicitly required to memorize the targets' identities.

\section{Loc ACC}

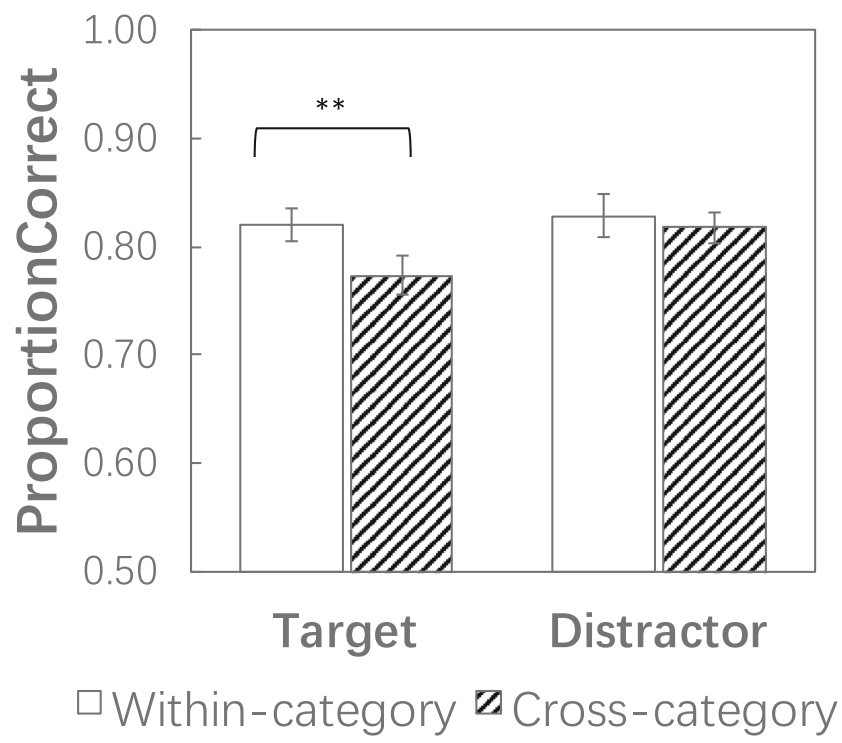

Fig. 4 Mean tracking performance $( \pm 1 S E)$ under the four conditions in Experiment 2
However, the effects persisted only for the targets and not for the distractors. We attributed this finding to attentional deployment. Previous studies have claimed that categorical perception of color arises from postperceptual processes, such as attention (He et al., 2014; Witzel \& Gegenfurtner, 2015), which is perfectly supported by the results of Experiment 2 . In the tracking task, the targets were located within the attentional foci, whereas generally the distractors were not. Since the targets in the distractor within-category condition and the distractor cross-category condition were both cross category, we could reasonably assume that no difference existed between them when attention was paid solely to the target colors. However, the occurrence of $\mathrm{CP}$ effects of the distractors in Experiment 1 suggests that Witzel and Gegenfurtner's (2015) argument does not always hold. We propose that attention to categorical distinction can boost $\mathrm{CP}$ effects but that $\mathrm{CP}$ effects can also arise from the preattention level under certain task requirements.

\section{General discussion}

The current study investigated CP effects in the attentive tracking task. In Experiment 1, in which the participants needed to track the targets' locations and memorize their color identities, we found that location tracking was facilitated when the targets or the distractors were from the same color category compared with when they were from two different categories. To assess whether the mandatory memorization of object identity was necessary for the effect of color categories on location tracking, we conducted Experiment 2, in which the participants only needed to track the targets' locations. Experiment 2 showed that the effects of the target color categories still occurred, but that the distractor within-category benefit no longer persisted. Altogether, our study suggested that the tracking procedure could produce $\mathrm{CP}$ effects and that the task requirement mattered. Different mechanisms may be responsible for the $\mathrm{CP}$ effects in the two experiments, leading to a discrepancy in the distractor CP effects.

The main result of our study was the presence of $\mathrm{CP}$ effects in tracking, which seemed to contradict the previous study suggesting noncategorical color perception during MOT (Sun et al., 2018). However, the experimental settings of the current study were distinct from those of the previous study. In the previous study, all of the targets shared one color and the distractors shared another, promoting automatic feature-based grouping and target-distractor segregation. The color categories of the objects provided no additional information that enabled the participants to better perform the task. In the current study, hue variance existed within both the targets and the distractors, and the overall perceptual difference between the targets and the distractors was relatively small. Thus, formation of automatic feature-based grouping was difficult. 
Furthermore, we required the participants to memorize each target's color identity in Experiment 1. As expected, the introduction of these higher level processes, including attention and working memory, produced CP effects.

Specifically, Experiment 1 revealed within-category advantages under both the target and the distractor conditions. The finding that the target $\mathrm{CP}$ effects were comparable to the distractor CP effects (no interaction between category and object) might suggest that the CP effects in Experiment 1 arose from the early stages and required minimal attention. We proposed that the effects could be associated with the representational bias of the object hues at perceptual level. Our analysis of the estimated hue difference demonstrated that the target hues were biased in working memory at the response stage, which might be a result of perceptual warping during tracking. According to previous studies, colors in both perception and working memory are biased toward category centers and away from category boundaries, and the degree of the bias relies on their relative positions within a color category (Bae, Olkkonen, Allred, \& Flombaum, 2015; Kelly \& Heit, 2017; Souza \& Skóra, 2017). Concerning our study, two types of color assignment existed. The first was B1, G1, G2, and G3 (one blue and three greens) in the two within-category conditions, corresponding to $200^{\circ}, 170^{\circ}, 140^{\circ}$, and $110^{\circ}$, and the second was B2, B1, G1, and G2 (two blues and two greens) in the two cross-category conditions, corresponding to $230^{\circ}, 200^{\circ}, 170^{\circ}$, and $140^{\circ}$, respectively. Since the bluegreen boundary lie between B1 and G1, B1 and G1 were probably biased away from the boundary and toward their own category centers. Consequently, the subjective difference between $\mathrm{G} 1$ and $\mathrm{G} 2$ was smaller than that between $\mathrm{G} 1$ and $\mathrm{B} 1$, leading to target $\mathrm{CP}$ effects. Additionally, the difference between $\mathrm{B} 1$ and $\mathrm{G} 3$ was represented as larger than that between B2 and G2, because B1 was closer to the boundary than B2 and thus biased away from it to a greater degree. Thus, distractor CP effects occurred. The explanation of color space warping in the preattention stages for the $\mathrm{CP}$ effects has been supported by event-related potential studies (Clifford, Holmes, Davies, \& Franklin, 2010; Forder, He, Franklin, \& Malmierca, 2017; Holmes, Franklin, Clifford, \& Davies, 2009). The warping may result from language factors. This claim was in favor of Lupyan's (2012) label-feedback hypothesis, which suggested that language exerted transient modulation of ongoing perceptual processing. The requirement for memorization of the target colors prompted the participants to label all of the objects in the visual scene, which altered their perceptual organization. The CP effects were important for nonattended items. Because greater stimulus uncertainty occurred outside of the attentional foci, the CP effects would be more greatly recruited as a means of reducing perceptual uncertainty (Bae et al., 2015; Forder et al., 2017; Regier \& Xu, 2017) from a computational perspective. Note that the 110 color (G1) is named "green" here, but its actual category membership was not assessed. It might be considered as nongreen (e.g. yellow) by some participants. It is possible that the within-category condition would be facilitated to a larger extent if the 110 color was named "yellow" than if it was named "green" because the categorical relationship between the target set and the distractor set was different under these two cases. It is an interesting question for future studies. However, the category membership (green or yellow) of the 110 color wound not comprise the current conclusion that color categories modulate location tracking performance.

By contrast, Experiment 2 observed target $\mathrm{CP}$ effects but not distractor CP effects. This finding had two implications. First, the role of the target color categories in tracking was distinct from that of the distractor color categories. Second, the pattern of the $\mathrm{CP}$ effects in Experiment 2 was different from our findings in Experiment 1. We attributed the difference in CP effects between the targets and the distractors to attentional deployment, which was consistent with Witzel and Gegenfurtner's (2015) opinion that CP effects occurred when participants paid attention to the categorical distinction. In Experiment 2, the targets shared the same category (green) under the target within-category condition, and the shared categorical code could be used by the participants to better group the targets. As a result, the target within-category condition achieved a better location tracking performance than the target cross-category condition. However, the targets were both cross category under the distractor within-category condition and the distractor cross-category condition and thus produced no CP effects for the distractors. Experiment 1 showed a different pattern of CP effects, probably because the demand to memorize the targets' color identities promoted early encoding of the objects' categorical information, which no longer required substantial attention to be processed and was able to affect location tracking. This hypothesis rebuts Witzel and Gegenfurtner's (2015) argument but is in need of more evidence, especially for early-stage color space warping. Techniques such as eye tracking or fMRI could be adopted in further investigations.

When reviewing studies of categorical color perception, we observed inconsistent results. Some studies found CP effects, but others did not (Brown, Lindsey, \& Guckes, 2011; Clifford et al., 2010; Franklin et al., 2008; He et al., 2014; Holmes et al., 2009; Lindsey et al., 2010; Pilling, Wiggett, Ozgen, \& Davies, 2003; Roberson \& Davidoff, 2000; Webster \& Kay, 2012; Witzel \& Gegenfurtner, 2015; Yokoi, Watanabe, \& Saida, 2012). Recently, some attempts have been made to interpret this inconsistency and suggest conditions that produce CP effects (Davidoff, 2015; Witzel, 2018). Witzel (2018) raised a fundamental problem concerning how these studies controlled stimuli. When researchers compare cross-category to within-category stimuli, the continuous, noncategorical differences between colors must be set as equivalent. Therefore, contradictory evidence may exist if these studies determine 
color differences at different levels of vision. According to a substantial investigation of sensitivity to color differences (Brown et al., 2011; Lindsey et al., 2010; Witzel \& Gegenfurtner, 2013, 2014, 2016, 2018), Witzel (2018) suggested that color categories were not inherent to the sensitivity of the second-stage mechanisms and that color categories did not warp color perception at the basic level of color discrimination. Instead, the observed CP effects were "high-level" effects as a result of attention and subjective evaluation, which were better called "categorical facilitation" (Witzel, 2018). We believe that the CP effects found in Experiment 2 are within the scope of Witzel's (2018) "high-level" effects, whereas the $\mathrm{CP}$ effects found in Experiment 1, especially the distractor CP effects, may suggest "low-level" effects. In other words, attention may be critical to encourage high-level CP effect, but $\mathrm{CP}$ effects can also be observed outside of the attentional foci. When the participants were required to process and memorize color identity, the categorical information could be coded automatically, leading to preattention $\mathrm{CP}$ effects. The findings of our study are relevant to the debate of cognitive penetrability. The results of Experiment 1 could be considered as evidence in favor of cognitive penetrability in that color categories shape how we perceive and segment moving colors. However, the percepts are not always penetrable, because the influence of color categories on tracking requires attention in Experiment 2.

Finally, a common concern for studies suggesting categorical effects is that perceptual color differences may not be well controlled using color spaces like CIELUV/CIELAB to equate color distance (He et al., 2014) or even different types of monitors to render stimuli (Zhang et al., 2018). In our study, the CIELAB color space was used to generate color stimuli which were presented on a calibrated CRT. If the perceived differences between cross-category and withincategory stimuli were not identical in our study, then we should also find a significant tracking difference between the distractor within-category and distractor cross-category conditions in Experiment 2. Moreover, the previous MOT study also adopted color stimuli from the CIELAB and failed to find any categorical effects (Sun et al., 2018). Hence, the CP effects found in the current study were unlikely to be due to failure to control the discriminability of the color stimuli. Future studies can make improvements when generating color stimuli.

\section{Conclusion}

The current study revealed color categorical effects in attentive tracking. Location tracking was facilitated when the targets or the distractors shared the same color category in a tracking task that required location tracking and color memorization at the same time, compared with when they were from two different categories (Experiment 1). By contrast, when location tracking was the sole demand and no explicit color processing was required, the target $\mathrm{CP}$ effects but not the distractor $\mathrm{CP}$ effects persisted (Experiment 2). We conclude here that color categories can dynamically modulate preattention visual processing when color working memory is involved but that attention is necessary to produce CP effects when color is irrelevant to the task.

Acknowledgments This study was supported by the General Program of National Natural Science Foundation of China (31271083) (to X.Z.) and the Key Program of National Natural Science Foundation of China (61632014) (to X.Z.).

\section{References}

Bae, G., Olkkonen, M., Allred, S. R., \& Flombaum, J. (2015). Why some colors appear more memorable than others: A model combining categories and particulars in color working memory. Journal of Experimental Psychology: General, 144(4), 744-763.

Brainard, D. H. (1997). The Psychophysics Toolbox. Spatial Vision, 10(4), 433-436.

Brown, A. M., Lindsey, D. T., \& Guckes, K. M. (2011). Color names, color categories, and color-cued visual search: Sometimes, color perception is not categorical. Journal of Vision, 11(12), 72-72.

Cavanagh, P., \& Alvarez, G. A. (2005). Tracking multiple targets with multifocal attention. Trends in Cognitive Sciences, 9(7), 349-354.

Clifford, A., Holmes, A., Davies, I. R., \& Franklin, A. (2010). Color categories affect pre-attentive color perception. Biological Psychology, 85(2), 275.

Daoutis, C., Pilling, M., \& Davies, I. R. L. (2006). Categorical effects in visual search for colour. Visual Cognition, 14(2), 217-240.

Davidoff, J. (2015). Color categorization across cultures. In A. Elliot, M. Farichild, \& A. Franklin (Eds.), Handbook of color psychology (pp. 259-278). New York, NY: Cambridge University Press. https://doi. org/10.1017/CBO9781107337930.013

Drivonikou, G. V., Kay, P., Regier, T., Ivry, R. B., Gilbert, A. L., Franklin, A., \& Davies, I. (2007). Further evidence that Whorfian effects are stronger in the right visual field than the left. Proceedings of the National Academy of Sciences of the United States of America, 104(3), 1097-1102.

Forder, L., He, X., Franklin, A., \& Malmierca, M. S. (2017). Colour categories are reflected in sensory stages of colour perception when stimulus issues are resolved. PLOS ONE, 12(5). https://doi.org/10. 1371/journal.pone. 0178097

Franklin, A., Clifford, A., Williamson, E., \& Davies, I. (2005). Color term knowledge does not affect categorical perception of color in toddlers. Journal of Experimental Child Psychology, 90(2), 114-141.

Franklin, A., Drivonikou, G. V., Clifford, A., Kay, P., Regier, T., \& Davies, I. R. L. (2008). Lateralization of categorical perception of color changes with color term acquisition. Proceedings of the National Academy of Sciences of the United States of America, 105(47), 18221-18225.

Gilbert, A. L., Regier, T., Kay, P., \& Ivry, R. B. (2006). Whorf hypothesis is supported in the right visual field but not the left. Proceedings of the National Academy of Sciences of the United States of America, 103(2), 489-494.

Hanley, J. R., \& Roberson, D. (2011). Categorical perception effects reflect differences in typicality on within-category trials. Psychonomic Bulletin \& Review, 18(2), 355-363. 
Harnad, S. (1987). Category induction and representation. Cambridge, UK: Cambridge University Press.

Harnad, S., Hanson, S., \& Lubin, J. (1991). Categorical perception and the evolution of supervised learning in neural nets. In D. W. Powers \& L. Reeker (Eds.), Working papers of the AAAI Spring Symposium on Machine Learning of Natural Language and Ontology (pp. 6574).

He, X., Witzel, C., Forder, L., Clifford, A., \& Franklin, A. (2014). Color categories only affect post-perceptual processes when same- and different-category colors are equally discriminable. Journal of the Optical Society of America. A: Optics, Image Science, and Vision, 31(4), 322-331.

Holmes, A., Franklin, A., Clifford, A., \& Davies, I. (2009). Neurophysiological evidence for categorical perception of color. Brain and Cognition, 69(2), 426-434.

Huang, L., Mo, L., \& Li, Y. (2012). Measuring the interrelations among multiple paradigms of visual attention: An individual differences approach. Journal of Experimental Psychology: Human Perception and Performance, 38(2), 414-428.

Kay, P., \& Kempton, W. (1984). What is the Sapir-Whorf hypothesis? American Anthropologist, 86(1), 65-79.

Kelly, L. J., \& Heit, E. (2017). Recognition memory for hue: Prototypical bias and the role of labeling. Journal of Experimental Psychology: Learning, Memory and Cognition, 43(6), 955-971.

Lindsey, D. T., Brown, A. M., Reijnen, E., Rich, A. N., Kuzmova, Y. I., \& Wolfe, J. M. (2010). Color channels, not color appearance or color categories, guide visual search for desaturated color targets. Psychological Sciience, 21(9), 1208-1214.

Lupyan, G. (2008). The conceptual grouping effect: Categories matter (and named categories matter more). Cognition, 108(2), 566-577.

Marsman, M., \& Wagenmakers, E.-J. (2017). Bayesian benefits with JASP. European Journal of Developmental Psychology, 14(5), $545-555$.

Meyerhoff, H. S., Papenmeier, F., \& Huff, M. (2017). Studying visual attention using the multiple object tracking paradigm: A tutorial review. Attention, Perception, \& Psychophysics, 79(5), 12551274. https://doi.org/10.3758/s13414-017-1338-1

Oksama, L., \& Hyona, J. (2008). Dynamic binding of identity and location information: A serial model of multiple identity tracking. Cognitive Psychology, 56(4), 237-283.

Pelli, D. G. (1997). The VideoToolbox software for visual psychophysics: Transforming numbers into movies. Spatial Vision, 10(4), 437-442.

Pilling, M., Wiggett, A. J., Ozgen, E., \& Davies, I. R. L. (2003). Is color "categorical perception" really perceptual? Memory \& Cognition, 31(4), 538-551.

Pylyshyn, Z., \& Storm, R. W. (1988). Tracking multiple independent targets: Evidence for a parallel tracking mechanism. Spatial Vision, 3(3), 179-197.

Regier, T., Kay, P., \& Khetarpal, N. (2007). Color naming reflects optimal partitions of color space. Proceedings of the National Academy of Sciences of the United States of America, 104(4), 1436-1441.

Regier, T., \& Xu, Y. (2017). The Sapir-Whorf hypothesis and inference under uncertainty. Wiley Interdisciplinary Reviews: Cognitive Science, 8(6), e1440.

Roberson, D., \& Davidoff, J. (2000). The categorical perception of colors and facial expressions: The effect of verbal interference. Memory \& Cognition, 28(6), 977.
Roberson, D., \& Hanley, J. R. (2009). Only half right: Comment on Regier \& Kay. Trends in Cognitive Sciences, 13(12), 500-501; author reply 501 .

Roberson, D., \& Hanley, J. R. (2010). Relatively speaking: An account of the relationship between language and thought in the color domain. In B. Malt \& P. Wolff (Eds.), Words and the mind: How words capture human experience. https://doi.org/10.1093/acprof:oso/ 9780195311129.003 .0010

Roberson, D., Pak, H., \& Hanley, J. R. (2008). Categorical perception of colour in the left and right visual field is verbally mediated: Evidence from Korean. Cognition, 107(2), 752-762.

Scholl, B. J. (2009). What have we learned about attention from multipleobject tracking (and vice versa)? In D. Dedrick \& L. Trick (Eds.), Computation, cognition, and Pylyshyn (pp. 49-77). Cambridge, MA: MIT Press..

Souza, A. S., \& Skóra, Z. (2017). The interplay of language and visual perception in working memory. Cognition, 166, 277.

Sun, M., Zhang, X., Fan, L., \& Hu, L. (2018). Hue distinctiveness overrides category in determining performance in multiple object tracking. Attention, Perception, \& Psychophysics, 80(2), 374-386. https://doi.org/10.3758/s13414-017-1466-7

Webster, M. A., \& Kay, P. (2012). Color categories and color appearance. Cognition, 122(3), 375-392.

Winawer, J., Witthoft, N., Frank, M. C., Wu, L., Wade, A., \& Boroditsky, L. (2007). Russian blues reveal effects of language on color discrimination. Proceedings of the National Academy of Sciences of the United States of America, 104(19), 7780-7785.

Witzel, C. (2018). Misconceptions about colour categories. Review of Philosophy and Psychology, 1-42. Advance online publication. https://doi.org/10.1007/s13164-018-0404-5

Witzel, C., \& Gegenfurtner, K. R. (2013). Categorical sensitivity to color differences. Journal of Vision, 13(7), 1-1.

Witzel, C., \& Gegenfurtner, K. R. (2014). Category effects on colour discrimination. In W. Anderson, C. Biggam, C. Hough, \& C. Kay (Eds.), Colour studies: A broad spectrum (pp. 200-211). Amsterdam, Netherlands: John Benjamins Publishing Company.

Witzel, C., \& Gegenfurtner, K. R. (2015). Categorical facilitation with equally discriminable colors. Journal of Vision, 15(8), 22.

Witzel, C., \& Gegenfurtner, K. R. (2016). Categorical perception for red and brown. Journal of Experimental Psychology: Human Perception and Performance, 42(4), 540.

Witzel, C., \& Gegenfurtner, K. R. (2018). Are red, yellow, green, and blue perceptual categories? Vision Research, 151, 152-163. https://doi. org/10.1016/j.visres.2018.04.002

Yokoi, K., Watanabe, K., \& Saida, S. (2012). Rapid and implicit effects of color category on visual search. Optical Review, 19(4), 276-281.

Zhang, GL., Li AS., Miao, CG., He, X., Zhang, M., Zhang, Y. (2018). A consumer-grade LCD monitor for precise visual stimulation. Behavior Research Methods 50(4):1496-1502.

Publisher's note Springer Nature remains neutral with regard to jurisdictional claims in published maps and institutional affiliations. 